\title{
Eckpfeiler erweiterte Produzentenverantwortung
}

\author{
Die erweiterte Produzentenverantwortung stellt ein wichtiges Leitprinzip für eine integrierte \\ Produktpolitik dar. Seit 1994 ist sie in Schweden gesetzlich verankert. Verordnungen regeln die \\ Nachgebrauchsphase von Verpackungen, Altpapier, Reifen, Autos und Elektronikprodukten. \\ Ökologische Erfolge sind sichtbar. Angesichts des bisher begrenzten Einflusses auf das Pro- \\ duktdesign ist darauf zu achten, dass weitere Anreize für Produktinnovationen gesetzt werden.
}

D Crik Westin und Nilsa Wabher, ie Erweiterte Produzentenverantwortung (extended producer responsibility, EPR) ist eine Strategie zur Verbesserung der Umwelteigenschaften von Produktsystemen. Sie zielt auf die Verantwortung von Produzenten während des gesamten Lebenszyklus von Produkten. Sie umfasst dabei insbesondere folgende Bestandteile (vgl. Abb. 1): lichkeiten der Verantwortungsausweitung, indem Hersteller aufgefordert werden, über die umweltrelevanten Eigenschaften der von ihnen angebotenen Produkte zu informieren.

Eine Situation, in der der Produzent während des gesamten Lebenszyklus Eigentümer des Produktes bleibt, illustriert die vollständige Umsetzung des Prinzips der erweiterten Produzentenverantwortung. Dies kann der Fall sein, wenn Produzenten Produktservicesysteme anbieten. Letztere werden

\begin{tabular}{|c|c|c|c|}
\hline \multicolumn{4}{|c|}{ Abb. l: Erweiterte Produzentenverantwortung } \\
\hline Lebenszyklus des Produktes & Produktions-/Transportphase & Nutzungsphase & Entsorgungsphase \\
\hline Art der Verantwortung & $\begin{array}{l}\text { - Arbeitsschutz } \\
\text { - Vermeidung und Management von } \\
\text { Umweltbelastungen aus Produktionsprozessen } \\
\text { - wirtschaftliche und rechtliche } \\
\text { Verantwortung für die umweltgerechte } \\
\text { Entsorgung von Industrieabfällen }\end{array}$ & $\begin{array}{l}\text { Zivilrechtliche Haftung } \\
\text { für gefährliche Produkte }\end{array}$ & $\begin{array}{l}\text { Wirtschaftliche } \\
\text { und/oder physische } \\
\text { Verantwortung für die } \\
\text { Entsorgung von } \\
\text { Altprodukten }\end{array}$ \\
\hline $\begin{array}{l}\text { Konventionelle Ver- } \\
\text { antwortungsaufteilung }\end{array}$ & & Produzenten und Handel & $\begin{array}{l}\text { Staatliche/kommu- } \\
\text { nale Einrichtungen }\end{array}$ \\
\hline $\begin{array}{l}\text { Im Rahmen der EPR } \\
\text { Quelle: Tojo, Naoko: Analy }\end{array}$ & Pro & $\begin{array}{l}\text { entenverantwortung } \\
\text { arative study of selected } \\
\text { iversität Lund, IlIEE Con }\end{array}$ & $\begin{array}{l}\text { EEE. } \\
10\end{array}$ \\
\hline
\end{tabular}

Haftung bezieht sich auf die gesetzlich festgelegte Verantwortung für nachweislich durch das fragliche Produkt verursachte Umweltschäden.

- Wirtschaftliche Verantwortung bedeutet, dass der Produzent die im Verlauf des Lebenszyklus entstehenden umweltschutzbezogenen Kosten ganz oder teilweise trägt - zum Beispiel für das Einsammeln, Recycling oder die endgültige Entsorgung des angebotenen Produkts.

- Physische Verantwortung charakterisiert solche Systeme, die die Hersteller in das eigentliche physische Entsorgungsmanagement einbeziehen.

- Wissens- und Informationsverantwortung beziehen sich auf mehrere unterschiedliche Mög- definiert als vorab konzipierte Systeme von Produkten, Dienstleistungen, unterstützender Infrastruktur und erforderlichen, zuvor errichteten Netzwerken zur Befriedigung von auf dem Markt bestehenden Verbraucherbedürfnissen (2). Die Förderung solcher Systeme kann es Produzenten erleichtern, die vom Produktsystem ausgehende Umweltbelastung zu verringern.

\section{Unterschiedliche EPR-Ansätze}

Bei der Einführung der EPR gibt es drei verschiedene Ansätze in Abhängigkeit von der Stärke und dem Ausmaß staatlicher Beteiligung:

1. Freiwillige Initiativen von Produzenten.
2. Im Falle einer gemeinsam ausgehandelten Vereinbarung unterzeichnen Produzenten und Regierungen beispielsweise einen Vertrag zur Erreichung bestimmter ökologischer Ziele wie etwa der Reduktion toxischer Produktbestandteile oder eines Produkt-Redesigns zugunsten von Haltbarkeit bzw. Wiederverwertbarkeit (3).

3. Der verpflichtende Ansatz etwa durch Vorschriften bedeutet, dass der Staat Anforderungen an entsprechende Akteure in der Gesellschaft stellt, die diese zu erfüllen haben. Die meisten Regierungen haben bei der Integration der Produzentenverantwortung in ihre Vorschriften drei Rahmenbedingungen vorgegeben:

- Präzise seitens der Hersteller zu erreichende Ziele und Zeitrahmen;

- Definition von Verantwortlichkeiten, welche die einzelnen Industriezweige übernehmen müssen, sowie Bedingungen, zu denen diese an unabhängige Organisationen übertragen werden können; und

- sonstige Maßnahmen, die für die politische Gesamtstrategie von Bedeutung sind.

Auch ökonomische Instrumente stellen eine verpflichtende Maßnahme dar, die bei der Entwicklung von Strategien der erweiterten Produzentenverantwortung eingesetzt wird. Laut OECD sind die in diesem Rahmen am häufigsten eingesetzten ökonomischen Instrumente Produktabgaben, Umweltsteuern, Entsorgungsgebühren und Pfandrückerstattungssysteme.

\section{Schwedische Erfahrungen mit Pflicht-EPR}

Gutachten belegen, dass die bestehende Gesetzgebung zur Produzentenverantwortung ein wichtiges Instrument zur Förderung der Altproduktsammlung sowie der gesteigerten Rückgewinnung und verminderten Deponierung von Müll darstellt. Verordnungen im Bereich der Produzentenverantwortung haben zur Reduktion von Abfall (Verpackungen) und dessen Deponierung (Verpakkungen, Altpapier, Reifen) beigetragen (4). Abb. 2 gibt hierzu schwedische Zahlen aus dem Jahr 1999.

Ab 2001 werden in Schweden neue Zielvorgaben für Verpackungen gelten. Bei mehreren Verpackungsarten wie Aluminium, Kunststoff, Papier und Pappe werden sich wahrscheinlich Schwierigkeiten bei der Erreichung der höheren Werte ergeben.

Neue Zielvorgaben existieren bereits für Altpapier (75 Prozent Materialrecycling 2000) und Autos 


\begin{tabular}{|l|c|c|}
\hline \multicolumn{2}{|c|}{ Abb. 2: Recydingergebnisse in Schweden $\mathbf{9 9 9}$} \\
\hline Produkt/ Material & Recycling-bzw. Wiederverwendungsanteil & Zielvorgaben 1999 \\
\hline Altpapier & $79 \%$ & - \\
\hline Reifen & $95-100 \% 1)$ & $80 \%$ \\
\hline Autos & $81 \% 2)$ & - \\
\hline Glasverpackungen & $84 \%$ & $70 \%$ \\
\hline Kunststoffverpackungen & $34 \%$ & $30 \%$ \\
\hline Papier- und Kartonverpackungen & $40 \%$ & $30 \%$ \\
\hline Wellpappenverpackungen & $84 \%$ & $65 \%$ \\
\hline Stahlverpackungen & $62 \%$ & $50 \%$ \\
\hline Aluminiumverpackungen & $33 \%$ & $50 \%$ \\
\hline Mehrweg-Glasbehälter & $98 \%$ & $95 \%$ \\
\hline Aluminium-Getränkedosen & $84 \%$ & $90 \%$ \\
\hline Mehrweg-PET-Flaschen & $91 \%$ & $90 \%$ \\
\hline
\end{tabular}

1) einschlieflich Energierückgewinnung, 2) Angabe basierend auf $24 \%$ aller Alffahrzeuge

Quelle: Swedish EPA: Have the producers reached the goals - a follow-up of the producer responsibility 1999 Report 5078 (in Swedish), Stockholm 2000

(85 Prozent Materialrecycling 2002). Auch für Holz und sonstige Verpackungen wird es Zielvorgaben geben.

Die Umwelteffekte von Rechtsvorschriften lassen sich im allgemeinen schwer bemessen. Aus Ökobilanzen geht hervor, dass Energiebedarf und Emissionen zurückgehen, wenn Verpackungsmaterialien recycelt oder wiederverwendet werden. Auch machen sie deutlich, dass die Deponierung die ökologisch deutlich schlechteste Alternative für sämtliche untersuchten Materialien darstellt. Die schwedische Umweltbehörde konnte nachweisen, dass Rechtsvorschriften maßgeblich zur Erreichung der Ziele nachhaltiger Abfallwirtschaft für die angegebenen Produktgruppen beigetragen haben (5).

In welchem Verhältnis die sich aus der Einführung der Produzentenverantwortung für Verpackungen ergebenden Grenzkosten der erhöhten Rückgewinnung zum Umweltnutzen stehen, wird noch diskutiert und in einer laufenden Studie von CIT Ecologik näher untersucht. Unter anderem bestehen unterschiedliche Ansichten darüber, wie die Bemühungen der Haushalte, Müll bereits an dessen Entstehungsort zu trennen, zu bewerten sind.

Die bestehende Rechtslage zur Produzentenverantwortung hatte bislang keinerlei schlüssig belegbare ungünstige Auswirkungen auf die Wettbewerbsfäbigkeit. Aus der Tatsache, dass einige Hersteller nicht an gemeinschaftlich organisierten Systemen beteiligt sind, können sich jedoch noch Probleme in diesem Bereich ergeben (6). Es ist wichtig, das Bewusstsein der Produzenten für den tieferen Sinn der Verantwortung zu schärfen und die Kontrolle zu verbessern, damit das Vertrauen in das System nicht untergraben wird.
Nach Ansicht der schwedischen Umweltbehörde ist die Einführung einer allgemeinen gesetzlichen Produzentenverantwortung für das Altproduktmanagement zur Zeit nicht gerechtfertigt. Eine Ausweitung der verpflichtenden Produzentenverantwortung auf das Altproduktmanagement sollte schrittweise durchgefuihrt werden. Eine unabhängige Studie soll bis Mitte 2001 hier nähere Vorschläge unterbreiten. Ein wichtiges Kriterium besteht darin, dass Produkte ein dringliches Problem im Abfallstrom darstellen, zum Beispiel aufgrund ihrer Gefährlichkeit (Batterien), Abfallmenge (Verpackungsmüll) oder beidem (elektrische/ elektronische Produkte, Fahrzeuge).

\section{EPR und Innovationen}

Erfahrungen mit der in Schweden bestehenden gesetzlichen Produzentenverantwortung belegen, dass der Anreiz zur Vermeidung produktbezogener Umweltbelastungen mittels verändertem Produktdesign durch einen neuen politischen Ansatz gefördert werden muss.

Ein Großteil der Umweltbelastungen vieler Produkte liegt bereits in deren Design begründet. Das größte Potenzial für produktbezogene Umweltverbesserungen besteht in entsprechenden Produktinnovationen. Integrierte Produktpolitik (IPP) sollte sich daher zukünftig nicht nur auf bereits bestehende Produkte, sondern auch auf zukünftige Produkte, also Produktinnovationen, konzentrieren (7).

\section{Der Innovationsgrad - ein Potenzial für Umweltverbesserungen}

Bei Produktinnovationen geht es darum, neue Produkte herzustellen, die auf dem Markt profita- bel verkauft werden können. Den Kern dieses Innovationsprozesses bildet die technologische Entwicklung. Allgemein wird zwischen zwei verschiedenen Stufen unterschieden (8):

- Inkrementelle Innovationen beziehen sich auf Produktverbesserungen und neue Produkte innerhalb bestehender technologischer Rahmenbedingungen - etwa die Herstellung eines neuen Automodells oder von Chips mit einer neuen Geschmacksrichtung.

- Grundlegende Innovationen basieren auf neuer Technologie und führen letztlich zur Entstehung neuer Industriezweige. Manch grundlegende Innovation, ein Beispiel sind Produkte der Informations- und Kommunikationstechnik, bewirkt sogar systemische Veränderungen innerhalb der Gesellschaft.

Inkrementelle Innovationen tragen wesentlich zum industriellen Produktivitätszuwachs bei, langfristig verbraucht sich dieses Potenzial bestehender Technologien jedoch, weshalb grundlegende Innovationen zur Erhaltung des Wirtschaftswachstums erforderlich sind. Ähnlich können inkrementelle Innovationen die Umweltverträglichkeit von Produkten wesentlich verbessern, auf lange Sicht und für umfangreichere Verbesserungen sind jedoch grundlegende Innovationen erforderlich. Beide Innovationsarten bergen ein Potenzial für umfangreiche Verbesserungen der ökologischen Leistung einer Gesellschaft und sollten daher von IPP thematisiert werden.

Eine staatliche Förderung grundlegender Innovationen und systemischer Veränderungen sowie inkrementeller Innovationen ist möglich - jedoch nur unter Einsatz verschiedener Instrumente wie etwa eines modernen öffentlichen Beschaffungswesens oder einer gezielten Forschungs- und Entwicklungsförderung. Ein Problem besteht hier darin, dass Unsicherheit und Nichtwissen in Bezug auf ihr Potenzial vorab um so größer sind, je radikaler die Innovation ist. Dies erschwert die Entwicklung der jeweiligen Strategien.

Mit grundlegenden Innovationen ist eine Abwendung von bestehenden Produkten verbunden sowie die Hinwendung zur Befriedigung neuer Bedürfnisse beziehungsweise alter Bedürfnisse auf grundlegend neue Art und Weise. Vielleicht wäre ein funktioneller Ansatz bei der Entwicklung solcher Strategien lohnender als ein produktbezogener.

\section{Gemeinsame Verantwortung}

Wer ist also verantwortlich für Innovationen, die umweltverträgliche Produkte nach sich ziehen? Die Verantwortung kann nicht nur dem Hersteller 
übertragen werden, der die neuen Produkte am Ende den Kunden anbietet. Eher muss sie von einer Gruppe unterschiedlicher Akteure übernommen werden. Dies deutet auf eine Politik hin, die sich nicht auf Produzenten im Sinne von Akteuren entlang der Wertschöpfungskette gründet, sondern eher auf einen weiten Kreis von Akteuren im Rahmen eines Innovationssystems. Die integrative IPPSichtweise sollte daher sämtliche Akteure von Innovationssystemen mitberücksichtigen, die Einfluss auf die Gestaltung neuer Produkte besitzen. Bedeutende Akteure sind Forschungseinrichtungen, Berater und Investoren.

Innovationen basieren auf Wechselwirkungen zwischen Menschen und Lernprozessen - Vorgängen, deren Steuerung weder einfach zu bewerkstelligen noch wünschenswert ist. Der Spielraum der Politik ist hier, durch Anreize innovative Aktivitäten in die gewünschte Richtung zu lenken.

Dies bedeutet für zukünftige Politik, dass Verantwortung als moralische oder gesellschaftliche Verantwortung gesehen werden sollte, die jeden von uns angeht. Wichtig ist, dass die Verantwortung für Innovationen, die zu umweltverträglicheren Produkten führen, nicht auf rechtliche Pflichten beschränkt ist und dass zukünftige Produktpolitik auch solche Instrumente einsetzt, die eher stimulieren als regulieren.

Forschungsergebnisse unterstreichen die Bedeutung flexibler Fristen im Rahmen der auf Innovationsstimulation abzielenden Politik (9). Die Bereitschaft von Firmen, ein Innovationsrisiko einzugehen, verringert sich bei Androhung von Strafe im Falle der nicht rechtzeitigen Beendigung eines Entwicklungsprojektes.

\section{Dynamischere Innovationssysteme durch Netzwerke}

Die Wahrnehmung ökologischer Anforderungen wird über die Netzwerke von Innovationssystemen vermittelt. In einem dynamischen Innovationssystem bedeutet Interaktion nicht nur Austausch kodifizierter Informationen. Die beteiligten Akteure entwickeln nämlich auch ein ergänzendes stillschweigendes Wissen, das sie in die Lage versetzt, die betreffenden Informationen verarbeiten $\mathrm{zu}$ können. Dies erfordert verstärkte Interaktionen. Intensivere Beziehungen sind daher wichtig für die Fähigkeit eines Innovationssystems, auf Umweltanforderungen reagieren zu können.

Ökologische Anforderungen des Staates oder privater Kunden können auch in wirtschaftliche oder funktionelle Begriffe übersetzt werden, weshalb die Kommunikation über Umweltfragen nicht immer von anderen Interaktionen zu trennen ist. Das Vermögen eines Innovationssystems, auf ökologische Anforderungen reagieren zu können, wird daher auch von seinem Vermögen abhängen, auf sonstige Ansprüche reagieren zu können. Die Errichtung und Förderung von Netzwerken stellt einen Schwerpunkt der Innovationspolitik dar. Innovationspolitik ist ein bedeutender komplementärer und unterstïtzender politischer Bereich der IPP.

\section{EPR als Teil schwedischer IPP}

In einer kürzlich ergangenen Mitteilung der schwedischen Regierung geht es um eine Strategie zur Einführung einer IPP in Schweden, innerhalb der EU und weltweit (10). Betont wird die Verantwortung sämtlicher Anspruchsgruppen für die Förderung von Nachhaltigkeit bei Produktion und Konsum. Sowohl administrative, materielle als auch marktgesteuerte Anreize und Initiativen auf Seiten der Industrie wie auch sonstiger Gruppen seien erforderlich. Es wird angeregt, dass das sechste Umweltaktionsprogramm der EU ein allgemeines Prinzip zur Minimierung der von Produkten ausgehenden Umweltbelastung enthalten sollte sowie einen Grundsatz, dass Produzenten für die von ihren Produkten ausgehende Umweltbelastung während des gesamten Lebenszyklus die Verantwortung übernehmen sollten.

Des weiteren verweist die schwedische Mitteilung auf die Verantwortung der Hersteller, Informationen über die Umweltverträglichkeit ihrer Produkte einzuholen und zur Verfuigung zu stellen. Ein Hersteller sollte kein Produkt auf den Markt bringen, ohne zu wissen, was es enthält, und ohne dessen ökologische Auswirkungen während des Produktlebenszyklus überprüft zu haben. Zulieferer und Hersteller sind am besten über die in ihren Produkten enthaltenen Materialien und Substanzen informiert und von daher am ehesten in der Lage, produktbezogene Umweltinformationen zu liefern. Hierfür sollten sie gemeinsam die Verantwortung übernehmen sowie dafür, die Informationen an unterschiedliche Zielgruppen anzupassen und sicherzustellen, dass sie leicht verständlich sind.

Nur wenige Produkte werden ausschließlich in Schweden hergestellt, benutzt und entsorgt. Produktion und Konsum Schwedens sind weitgehend in einen Verbund integriert und abhängig von Produktion und Konsum in anderen Ländern. Der schwedische Staat unterstïtzt daher nachdrücklich die Entwicklung einer EU-weiten IPP mit dem Hauptziel der Einführung einheitlicher Regeln und gemeinsamer Maßnahmen, die für eine umfassende Verringerung der Umweltbelastungen durch Produkte unumgänglich sind. Ein großes Verbesserungspotenzial besteht darin, sich ganz am Anfang des Produktentwicklungsprozesses, der Innovationsphase, auf Umweltaspekte zu konzentrieren und diese zu integrieren. IPP im Rahmen der EU sollte daher dazu beitragen, eine gemeinsame Plattform für Umwelt- und Innovationspolitik zu schaffen.

\section{Anmerkungen}

(1) Lindhqvist, T.: What is Extended Producer Responsibility? International Seminar in Lund arranged by the Swedish Waste Research Council. Lund 1998.

(2) Swedish Enviromental Protection Agency: Product Service Systems, AFR-report 288, Stockholm 2000.

(3) Swedish EPA: Environmental agreements - a possibility in the environmental work. Report 5064 (in Swedish),

Stockholm 2000

(4) Swedish EPA: Producers' responsibility for environmental impacts from products. Report 5043 (in Swedish), Stockholm 1999.

(5) Swedish EPA: Extended producer responsibility for pakkaging - what is in it for the environment? Report 4938 (in Swedish), Stockholm 1998.

(6) Swedish Competition Authority, National Board of Trade, National Board for Technical and Industrial Development:

The Environment, Trade and Competition - playing rules for efficient markets. Stockholm 1999.

(7) Ahlner, Eva/ Markusson, Nils: Current and future producer responsibility in Sweden. Paper presented at the IPTS/ ESTO Workshop on making environmentally sound products competitive - a focus for IPP. Stockholm 2000.

(8) Freeman, Chris: The nature of innovation and the evolution of the productive system. In: OECD (ed.): Technology and Productivity. Paris 1991

(9) National Board for Technical and Industrial Development and Swedish National Energy Administration: Drivers of environmental innovation. Stockholm (forthcoming).

(10) The Swedish Government: A Strategy for an Environmentally Sound Product Policy. Government Communication 1999/2000, Nr. 114. Stockholm 2000.

(Übersetzung: Irene Siebcke)

Die Autorlnnen
Eva Ahlner und Erik Westin arbeiten als Principal
Technical Officers bei der Schwedischen Umweltbehörde.
Kontakt: Swedish EPA, Unit for sustainable industry
and trade, S-10648 Stockholm. Tel. 0046-8-698-
1458/ -1620, Fax -1253, E-mail: eah@environ.se,
ewe@environ.se
Nils Markusson arbeitet bei NUTEK - Swedish Board
for Industrial and Technical Development.
Kontakt: NUTEK, S-11786 Stockholm,
Tel. 0046-8-681-6648, Fax -9280,
E-mail: nils.markusson@nutek.se


(c) 20I0 Authors; licensee IÖW and oekom verlag. This is an article distributed under the terms of the Creative Commons Attribution Non-Commercial No Derivates License (http://creativecommons.org/licenses/by-nc-nd/3.o/), which permits unrestricted use, distribution, and reproduction in any medium, provided the original work is properly cited. 